\title{
Memristor-Based Reactance-Less Oscillator
}

\author{
M. Affan Zidan, Hesham Omran, A. G. Radwan and K. N. Salama
}

In this letter, the first reactance-less oscillator is introduced. By using memristor, the oscillator can be fully implemented on-chip without the need of any capacitors or inductors, which results in an area-efficient fully integrated solution. The concept of operation of the proposed oscillator is explained and detailed mathematical analysis is introduced. Closed-form expressions for the oscillation frequency and oscillation conditions are derived. Finally, the derived equations are verified with circuit simulations showing excellent agreement.

Introduction: Memristor, the fourth basic circuit element, was first proposed by L. Chua in 1971 [1], but it was not until 2008 when a passive physical implementation was realized [2]. Since then, memristor attracted researchers from multiple disciplines with its unique characteristics and showed potential importance in many fields, including non-volatile memory, reconfigurable logic and circuit design $[3,4,5]$.

In this letter, we introduce a reactance-less memristor-based oscillator. The increase/decrease of memristor resistance according to the applied voltage resembles the charging/discharging of a reactive element. The inherent delay in the memristor response is exploited to realize the oscillator function. The "resistance-storage" property of memristor eliminates the need for an energy-storing reactive element, i.e., capacitor or inductor. It should be noted that even ring oscillators are formed of delay stages which depend on the charging/discharging of intrinsic and extrinsic capacitances.

Using the mathematical model of HP memristor in [6], the memristor resistance as a function of time is given by

$$
R_{m}^{2}(t)=R_{o}^{2} \pm 2 k^{\prime} \int_{o}^{t} V_{m}(\tau) d \tau
$$

where $R_{m}$ is the memristor resistance, $R_{o}$ is the initial resistance of the memristor, $V_{m}$ is the voltage across the memristor, and $k^{\prime}=\mu_{v} R_{o n}\left(R_{o f f}-R_{o n}\right) / d^{2}$, where $\mu_{v}$ is the dopant 
drift mobility, $R_{o n}$ and $R_{\text {off }}$ are the minimum and maximum memristor resistances respectively, and $d$ is the length of the device. The dopant drift mobility is the physical limit that determines the response time of the memristor. For current values of $\mu_{v}$, the proposed oscillator is suitable for low frequency applications. However, the introduced concept is general and can be extended to higher frequencies given that technology advancement improves the response speed. Low frequency oscillators find usage in biomedical applications and embedded systems [7, 8]. However, off-chip components are usually used because large capacitances are required [7]. In [8], a novel technique was used to implement the oscillator on-chip, but the capacitor consumed $77.8 \%$ of the total chip area. By using memristor, the implementation proposed in this letter eliminates the need for capacitors or inductors allowing a fully integrated implementation in a very small area.

Proposed Circuit: The proposed circuit is based on a voltage divider between a resistor and a memristor, and a feedback function $\left(F\left(V_{i}\right)\right)$ as shown in Fig. 1 and Fig. 2. The voltage on the memristor is given by

$$
V_{i}(t)=V_{o}(t) \frac{R_{m}}{R_{m}+R_{a}}
$$

The memristor is connected in a polarity such that $R_{m}$ increases for positive $V_{i}$ and decreases for negative $V_{i}$. The threshold voltages $V_{p}$ and $V_{n}$ should be selected such that $V_{n}<0<V_{p}$. A simple implementation of $F\left(V_{i}\right)$ using two comparators and an AND gate is shown in Fig. 1, while other implementations are also possible.

Using Fig. 2, the operation of the oscillator can be traced assuming that we start at ' $a$ ':

$\mathrm{a} \rightarrow \mathrm{b}$ : At 'a' a positive voltage is applied on the memristor since $V_{o}=V_{o h}$. The memristor resistance will increase, and so will $V_{i}$ until the operating point reaches 'b'.

$\mathrm{b} \rightarrow \mathrm{c}$ : At 'b' the value of $V_{i}$ will just pass $V_{p}$, thus $V_{o}$ will switch to $V_{o l}$, and the operating point will jump to 'c'.

$c \rightarrow d$ : At ' $c$ ' a negative voltage is applied on the memristor. The memristor resistance will decrease, and so will $\left|V_{i}\right|$ until the operating point reaches 'd'. 


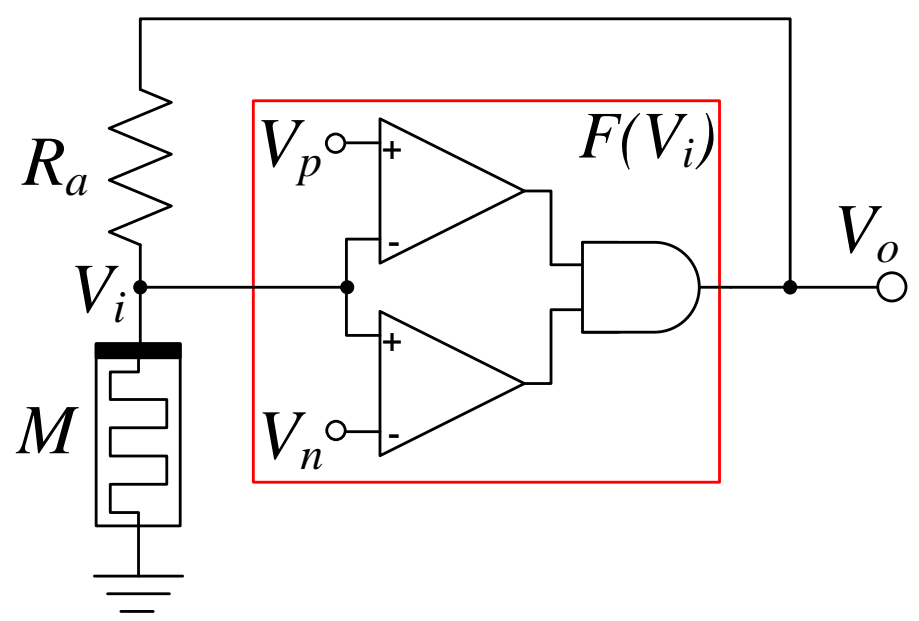

Figure 1: The proposed memristor-based reactance-less oscillator.

$\mathrm{d} \rightarrow \mathrm{a}$ : At 'd' the value of $V_{i}$ will just pass $V_{n}$, thus $V_{o}$ will switch to $V_{o h}$, and the operating point will jump to ' $a$ '.

The circuit will oscillate independent of the initial memristor resistance $\left(R_{o}\right)$. If the initial point is at $\left(V_{i}<V_{n}\right)$ the memristor resistance will decrease until reaching 'd'. If it starts at $\left(0<V_{i}<V_{p}\right)$ the memristor resistance will increase until reaching 'b'. In both cases, the circuit will start oscillation from 'b' or ' $d$ ' and will follow the path described previously. The cases $\left(V_{n}<V_{i}<0\right)$ and $\left(V_{i}>V_{p}\right)$ will not happen since $V_{i}$ and $V_{o}$ must have the same polarity.

Oscillation Condition: Based on (2), there is a single equivalent memristor resistance for a

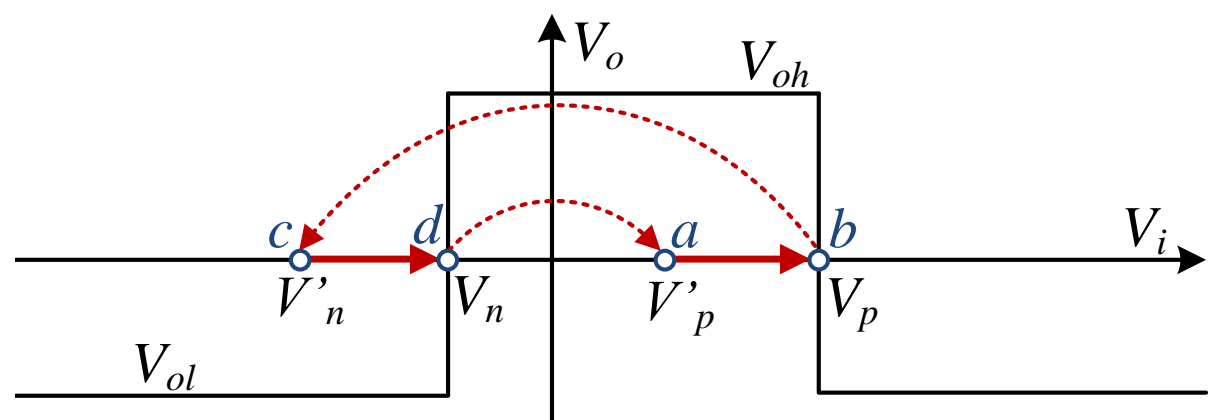

Figure 2: Transfer function of $F\left(V_{i}\right)$ showing transition between different operating points. 
given value of $V_{i}$. The resistances at $V_{i}=V_{p}$ and $V_{i}=V_{n}$ are given by

$$
R_{m p}=R_{a} \frac{V_{p}}{V_{o h}-V_{p}}, R_{m n}=R_{a} \frac{V_{n}}{V_{o l}-V_{n}}
$$

$R_{m p}$ and $R_{m n}$ have to be selected such that $R_{o n}<R_{m n}<R_{m p}<R_{o f f}$. Based on the circuit tracing given in the previous section, the oscillation will occur if $V_{p}$ and $V_{n}$ are selected such that

$$
V_{p}-V_{n} \frac{V_{o h}}{V_{o l}}>0
$$

Oscillation Frequency: The time required by the circuit to change its state from ' $a$ ' to ' $b$ ' is determined by the time required for the memristor to change its resistance from $R_{m n}$ to $R_{m p}$. The derivative of (1) with respect to time is given by

$$
R_{m} d R_{m}=k^{\prime} V_{i}(t) d t
$$

By substituting (2) into (5) then integrating, the equation can be written as

$$
\int_{0}^{T_{H}} d t=\frac{1}{k^{\prime} V_{o h}} \int_{R_{m n}}^{R_{m p}}\left(R_{m}+R_{a}\right) d R_{m}
$$

By solving the integration, the time of the positive half cycle is

$$
T_{H}=\frac{R_{m p}^{2}-R_{m n}^{2}+2 R_{a}\left(R_{m p}-R_{m n}\right)}{2 k^{\prime} V_{o h}}
$$

Similarly, the time of the negative half cycle is

$$
T_{L}=\frac{R_{m n}^{2}-R_{m p}^{2}+2 R_{a}\left(R_{m n}-R_{m p}\right)}{2 k^{\prime} V_{o l}}
$$

The duty cycle expression can be derived in a simple form by substituting (3) into (7) and (8)

$$
D=\frac{\left|V_{o l}\right|}{V_{o h}-V_{o l}}
$$

Using the same substitutions and after simplification, the frequency of oscillation is given 
by

$$
f_{o}=\frac{2 D k^{\prime} V_{o h}\left(V_{o h}-V_{p}\right)^{2}\left(V_{n}-V_{o l}\right)^{2}}{R_{a}^{2}\left(V_{p} V_{o l}-V_{n} V_{o h}\right)\left(2 V_{o h} V_{o l}-V_{o l} V_{p}-V_{o h} V_{n}\right)}
$$

Circuit Simulation: As the access to the experimental realization of memristor is very limited, researchers resort to SPICE and behavioral models of HP memristor [9], or emulate the memristor model using active circuitry [3]. The proposed oscillator was simulated using both SPICE and Verilog-A models, both giving similar results. We chose $R_{o n}, R_{o f f}, d$, and $\mu_{v}$ to be $100 \Omega, 38 \mathrm{k} \Omega, 10 \mathrm{~nm}$, and $10^{-10} \mathrm{~cm}^{2} \mathrm{~s}^{-1} V^{-1}$ respectively. Fig. 3 shows the transient simulation results. The memristor resistance oscillates between $R_{m n}$ and $R_{m p}$, which defines the location of the operating points. By substituting the circuit parameters into (3) and (10): $R_{m n}=3 k \Omega, R_{m p}=9 k \Omega$, and $f_{o}=3.51 \mathrm{~Hz}$, which shows excellent match to the simulation results. For further verification of the mathematical analysis presented, the oscillation frequency was tuned by sweeping $R_{a}$ from $2 k \Omega$ to $12 k \Omega$. The maximum error was $2.13 \%$ and $0.1 \%$ for SPICE and Verilog-A simulations respectively.
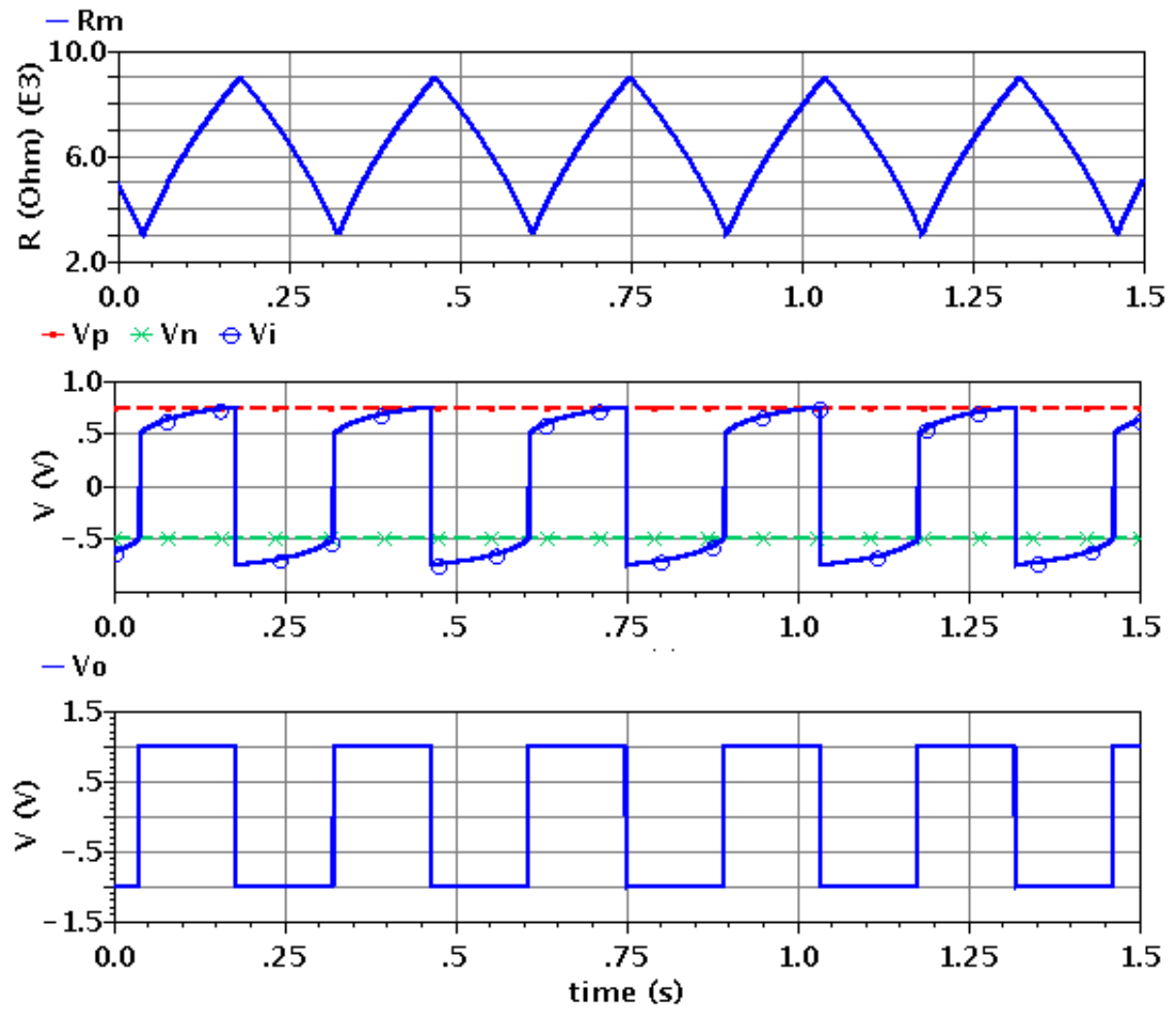

Figure 3: Transient simulation results for $R_{m}$ (upper), $V_{i}$ (middle), and $V_{o}$ (lower), for $R_{a}=$ $3 k \Omega, V_{o h}=1 \mathrm{~V}, V_{o l}=-1 \mathrm{~V}, V_{p}=0.75 \mathrm{~V}$, and $V_{n}=-0.5 \mathrm{~V}$. 
Conclusion: A memristor-based oscillator without using any capacitors or inductors was presented. The introduced reactance-less oscillator enables an area efficient implementation for low frequency oscillators, which find applications in biomedical and embedded systems. Mathematical analysis for the new oscillator is presented and verified using circuit simulation.

\section{References}

[1] Chua L. Memristor-The missing circuit element. IEEE Transactions on Circuit Theory. 1971;18(5):507-519.

[2] Strukov DB, Snider GS, Stewart DR. The missing memristor found. Nature. 2008;435:80-83.

[3] Pershin YV, Di Ventra M. Memristive circuits simulate memcapacitors and meminductors. Electronics Letters. 2010 1;46(7):517 -518.

[4] Witrisal K. Memristor-based stored-reference receiver - the UWB solution? Electronics Letters. 2009 2;45(14):713 -714.

[5] Talukdar A, Radwan AG, Salama KN. Generalized model for Memristor-based Wien family oscillators. Microelectronics Journal. 2011;42(9):1032 - 1038.

[6] Radwan AG, Zidan MA, Salama KN. HP memristor mathematical model for periodic signals and DC. In: IEEE International Midwest Symposium on Circuits and Systems (MWSCAS'10); 2010. p. 861-864.

[7] Elwakil AS, Ozoguz S. A low frequency oscillator structure. In: European Conference on Circuit Theory and Design (ECCTD’09); 2009. p. 588 -590.

[8] Hwang C, Bibyk S, Ismail M, Lohiser B. A very low frequency, micropower, low voltage CMOS oscillator for noncardiac pacemakers. IEEE Transactions on Circuits and Systems I: Fundamental Theory and Applications. 1995;42(11):962-966. 
[9] Biolek Z, Biolek D, Biolkova V. SPICE Model of Memristor with Nonlinear Dopant Drift. Radioengineering. 2009;18(2):210-214.

\section{Authors' affiliations:}

M. Affan Zidan, Hesham Omran, A. G. Radwan and K. N. Salama (Electrical Engineering Program, King Abdullah University of Science and Technology (KAUST), Thuwal, 239556900, Saudi Arabia)

E-mail: mohammed.zidan@kaust.edu.sa 